\title{
Review of Mechanisms of Release of Commonly Prescribed Tetracyclines
}

Pearl Kwong MD, PhD, FAAD a , Hilary Baldwin MD, FAAD ${ }^{b}$, Debbie Glaab MSN, CPNP-AC ${ }^{c}$, Rhonda Schreiber MS, RN c, Emma Hignett BS ${ }^{d}$

INTRODUCTION

Dermatologists prescribe more oral antibiotics than any other (a) 政 ${ }^{2}$ Since then there has been increased use of three newer eneration tetracyclines. Doxycycline was introduced in 1967, minocycline in 1971, and most recently sarecycline in 2018,2,11 These are available in various mechanisms of release (MOR). The most common MORs used in dermatology are immediate release (IR) delayed release (DR), and extended release (ER). While clinician prescribe all these MORs, there is a lack of synthesized informatio regarding their impact on clinical considerations for use. The data presented here is intended to increase clinician understanding of

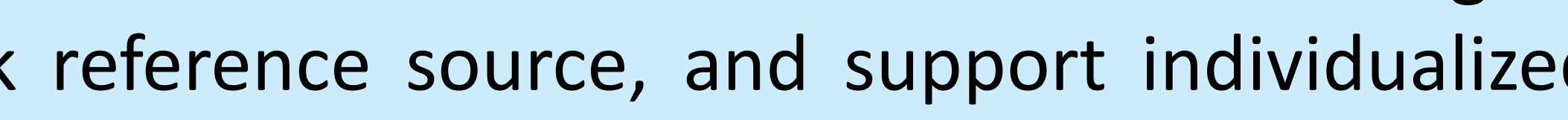
patient care.

\section{OBJECTIVE}

To create a resource regarding the MOR of oral tetracyclines used in dermatology.

\section{METHODS}

A systematic literature search of peer-reviewed publications was conducted over a 25-year period

- The primary focus of the review was to summarize differences between IR, ER, and DR and examine them pharmacologically and clinically

Data was summarized into tabular format for ease of reference

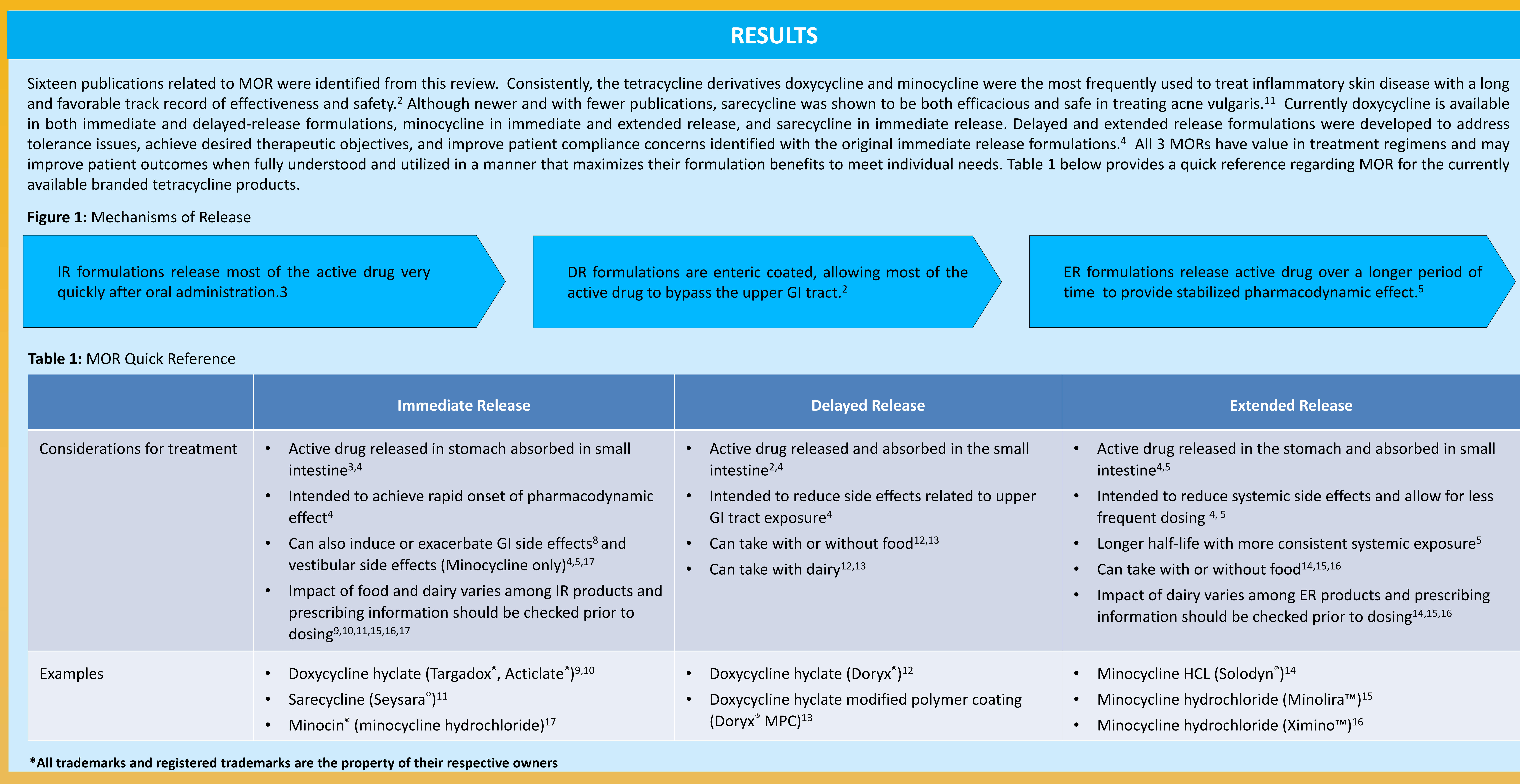

CONCLUSIONS

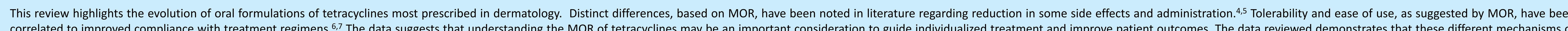
release have unique side effect profiles and considerations for treatment which may impact tolerability, patient preference, and compliance with treatment regimens. ${ }^{8}$

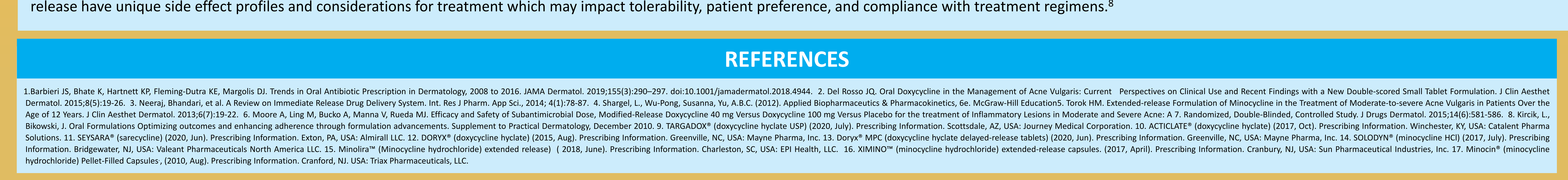

AFFILIATIONS

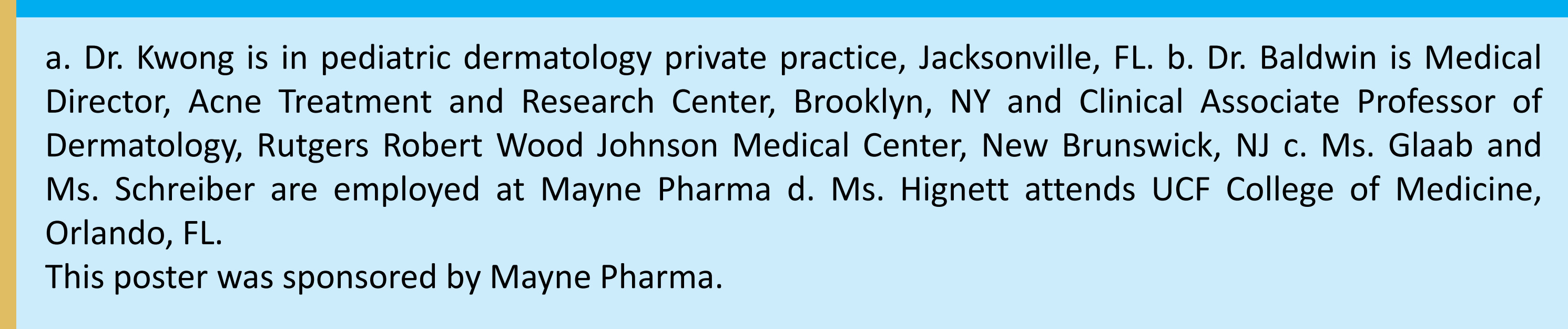




\section{Review of Mechanisms of Release of Commonly Prescribed Tetracyclines}

Pearl Kwong MD, PhD, FAAD a , Hilary Baldwin MD, FAAD ${ }^{b}$, Debbie Glaab MSN, CPNP-AC ${ }^{c}$, Rhonda Schreiber MS, RN c, Emma Hignett BS ${ }^{d}$

\section{INTRODUCTION}

Dermatologists prescribe more oral antibiotics than any other specialty. From the mid-19s0s to the early 1970 , the predominant

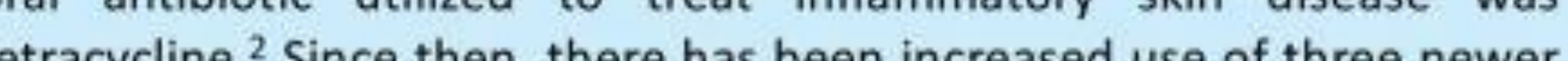
eneration tetracyclines. Doxycycline was introduced in 1967 minocline in 1971, and most recently sarecycline in 2018.,11 These e availabe in various mechanisms of release (MOR). The most common MORs used in dermatology are immediate release (IR) delayed release (DR), and extended release (ER). While clinicians prescribe all these MORs, there is a lack of synthesized information regarding their impact on clinical considerations for use. The data presented here is intended to increase clinician understanding of MORs, provide a quick reference source, and support individualized patient care.

\section{OBJECTIVE}

To create a resource regarding the MOR of oral tetracyclines used in dermatology.

METHODS

METHODS
- A systematic literature search of peer-reviewed publications was
conducted over a $25-$ year period
- The primary focus of the review was to summarize differences
between IR, ER, and DR and examine them pharmacologically and
clinically
- Data was summarized into tabular format for ease of reference

\section{RESULTS}

Sixteen publications related to MOR were identified from this review. Consistently, the tetracycline derivatives doxycycline and minocycline were the most frequently used to treat inflammatory skin disease with a long and favorable track record of effectiveness and safety. ${ }^{2}$ Although newer and with fewer publications, sarecycline was shown to be both efficacious and safe in treating acne vulgaris. ${ }^{11}$ Currently doxycycline is available in both immediate and delayed-release fom a lons, tolerance issues, achieve desired therapeutic objectives, and improve patient compliance concerns identified with the original immediate release formulations." All 3 MORs have value in treatment regimens and may available branded tetracycline products. Figure 1: Mechanisms of Release

IR formulations release most of the active drug very quickly after oral administration. 3 DR formulations are enteric coated, allowing most of the active drug to bypass the upper GI tract.
ER formulations release active drug over a longer period of time to provide stabilized pharmacodynamic effect. ${ }^{5}$

\section{Table 1: MOR Quick Reference}

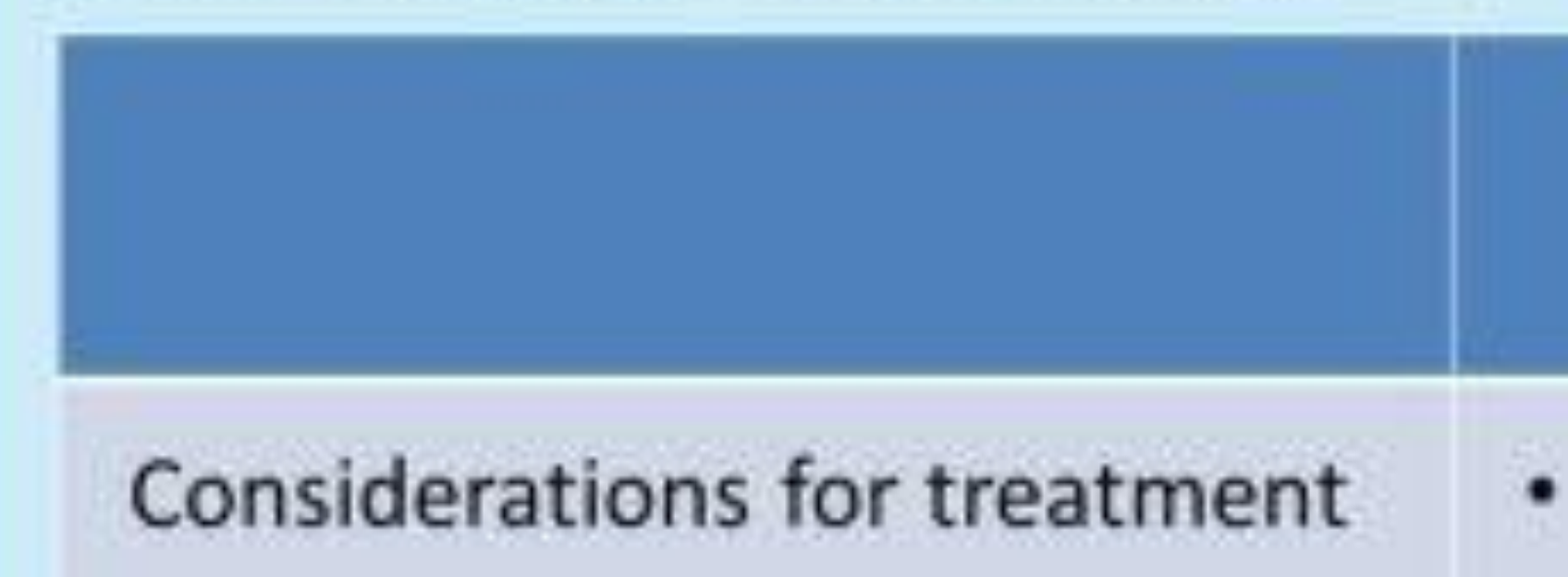

Immediate Release
- Active drug released in stomach absorbed in small
intestine ${ }^{3,4}$
- Intended to achieve rapid onset of pharmacodynamic
effect $^{4}$
- Can also induce or exacerbate $\mathrm{Gl}$ side effects ${ }^{8}$ and
vestibular side effects (Minocycline only)
- Impact, of food and dairy varies among IR products and
prescribing information should be checked prior to
dosing
- Doxycycline hyclate (Targadox , Acticlate $\left.{ }^{09}\right)^{9,10}$
- Sarecycline (Seysara")
- Minocin" (minocycline hydrochloride)

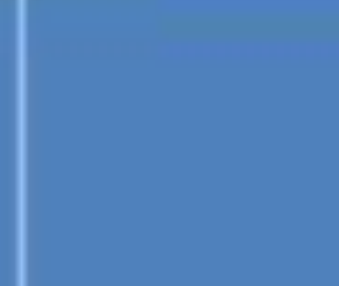

Delayed Release Active drug released and absorbed in the small intestine $e^{2,4}$

- Intended to reduce side effects related to upper GI tract exposure ${ }^{4}$

- Can take with or without food ${ }^{12,13}$

Can take with dairy $y^{12,13}$

- Doxycycline hyclate (Doryx $\left.)^{0}\right)^{12}$

Examples Doxycycline hyclate modified polymer coatin $\left(\text { Doryx }{ }^{*} \mathrm{MPC}\right)^{13}$

\section{Extended Release}

Active drug released in the stomach and absorbed in sma intestine 4.5

Intended to reduce systemic side effects and allow for les frequent dosing 4,5

Longer half-life with more consistent systemic exposure ${ }^{5}$ Can take with or without food ${ }^{14,15,16}$

- Impact of dairy varies among ER products and prescribing information should be checked prior to dosing ${ }^{14,15,16}$

Minocycline HCL (Solodyn") $)^{14}$

Minocycline hydrochloride (Minoliram ${ }^{15}$

- Minocycline hydrochloride (Minoliram $\left.{ }^{T+}\right)^{15}$

*All trademarks and registered trademarks are the property of their respective owners

\section{CONCLUSIONS}

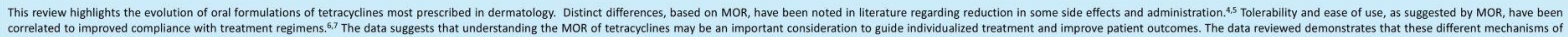
release have unique side effect profiles and considerations for treatment which may impact tolerability, patient preference, and compliance with treatment regimens.

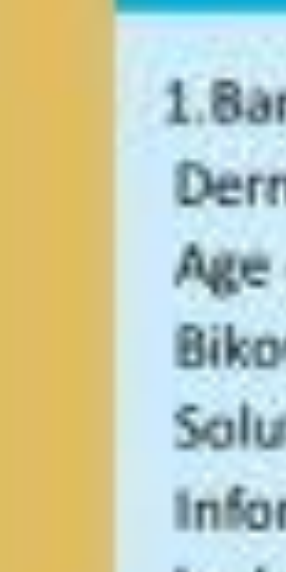

REFERENCES 